\title{
OPTIMIZING DATA-CENTER TCO WITH SCALE-OUT PROCESSORS
}

PERFORMANCE AND TOTAL COST OF OWNERSHIP (TCO) ARE KEY OPTIMIZATION METRICS

IN LARGE-SCALE DATA CENTERS. ACCORDING TO THESE METRICS, DATA CENTERS

DESIGNED WITH CONVENTIONAL SERVER PROCESSORS ARE INEFFICIENT. RECENTLY

INTRODUCED PROCESSORS BASED ON LOW-POWER CORES CAN IMPROVE BOTH

THROUGHPUT AND ENERGY EFFICIENCY COMPARED TO CONVENTIONAL SERVER CHIPS.

HOWEVER, A SPECIAlized SCALE-OUt PROCESSOR (SOP) ARCHITECTURE MAXIMIZES

ON-CHIP COMPUTING DENSITY TO DELIVER THE HIGHEST PERFORMANCE PER TCO AND

PERFORMANCE PER WATT AT THE DATA-CENTER LEVEL.

Boris Grot

EPFL

Damien Hardy

University of Cyprus

\section{Pejman Lotfi-Kamran}

Babak Falsafi

EPFL

Chrysostomos Nicopoulos

Yiannakis Sazeides

University of Cyprus
- ... We are in the midst of an information revolution, driven by ubiquitous access to vast data stores via a variety of richly networked platforms. Data centers are the workhorses powering this revolution. Companies leading the transformation to the digital universe, such as Google, Microsoft, and Facebook, rely on networks of megascale data centers to provide search, social connectivity, media streaming, and a growing number of other offerings to large, distributed audiences. A scale-out data center powering cloud services can house tens of thousands of servers that are necessary for high scalability, availability, and resilience. ${ }^{1}$

The massive scale of such data centers requires an enormous capital outlay for infrastructure and hardware, often exceeding $\$ 100$ million per data center. ${ }^{2}$ Similarly expansive are the power requirements, typically in the range of 5 to $15 \mathrm{MW}$ per data center, totaling millions of dollars in annual operating costs. With demand for information services skyrocketing around the globe, efficiency has become a paramount concern in the design and operation of large-scale data centers.

To reduce infrastructure, hardware, and energy costs, data-center operators target high computing density and power efficiency. Total cost of ownership (TCO) is an optimization metric that considers the costs of real estate, power delivery and cooling infrastructure, hardware acquisition costs, and operating expenses. Because server acquisition and power costs constitute the two largest TCO components, ${ }^{3}$ servers present a prime optimization target in the quest for more efficient data centers. In addition to cost, performance is also critical in scaleout data centers designed to service thousands of concurrent requests with real-time constraints. The ratio of performance to TCO (performance per dollar of ownership expense) is thus an appropriate metric for evaluating different data-center designs.

Scale-out workloads prevalent in largescale data centers rely on in-memory 


\begin{tabular}{|c|c|c|c|c|c|c|c|c|}
\hline \multirow[b]{2}{*}{ Processor } & \multicolumn{7}{|c|}{$\begin{array}{l}\text { Table 1. Server chip characteristics. The first three processors are existing designs, } \\
\text { and the last two processors are proposed designs. }\end{array}$} & \multirow[b]{2}{*}{$\begin{array}{l}\text { Cost per } \\
\text { processor } \\
\text { (\$) }\end{array}$} \\
\hline & Type & $\begin{array}{l}\text { Cores, } \\
\text { threads }\end{array}$ & $\begin{array}{c}\text { Last-level } \\
\text { cache size } \\
\text { (Mbytes) }\end{array}$ & $\begin{array}{l}\text { DDR3 } \\
\text { interfaces }\end{array}$ & $\begin{array}{l}\text { Frequency } \\
\text { (GHz) }\end{array}$ & $\begin{array}{l}\text { Power } \\
\text { (W) }\end{array}$ & $\begin{array}{c}\text { Area } \\
\left(\mathbf{m m}^{2}\right)\end{array}$ & \\
\hline $\begin{array}{l}\text { Big core, } \\
\text { big chip }\end{array}$ & Conventional & 6,12 & 12 & 3 & 3 & 95 & 233 & 800 \\
\hline $\begin{array}{l}\text { Small core, } \\
\text { small chip }\end{array}$ & Small chip & 4,4 & 4 & 1 & 1.5 & 6 & 62 & 95 \\
\hline $\begin{array}{l}\text { Small core, } \\
\text { big chip }\end{array}$ & Tiled & 36,36 & 9 & 2 & 1.5 & 28 & 132 & 300 \\
\hline $\begin{array}{l}\text { Scale-out, } \\
\text { in order }\end{array}$ & $\begin{array}{l}\text { Scale-Out } \\
\text { Processor }\end{array}$ & 48,48 & 4 & 3 & 1.5 & 34 & 132 & 320 \\
\hline $\begin{array}{l}\text { Scale-out, } \\
\text { out of order }\end{array}$ & $\begin{array}{l}\text { Scale-Out } \\
\text { Processor }\end{array}$ & 16,16 & 4 & 2 & 2 & 33 & 132 & 320 \\
\hline
\end{tabular}

processing and massive parallelism to guarantee low response latency and high throughput. Although processors ultimately determine a server's performance characteristics, they contribute just a fraction of the overall purchase price and power burden in a server node. Memory, disk, networking equipment, power provisioning, and cooling all contribute substantially to acquisition and operating costs. Moreover, these components are less energy proportional than modern processors, meaning their power requirements don't scale down well as the server load drops. Thus, maximizing the benefit from the TCO investment requires getting high utilization from the entire server, not just the processor.

To achieve high server utilization, data centers must employ processors that can fully leverage the available bandwidth to memory and I/O. Conventional server processors use powerful cores designed for a broad range of workloads, including scientific, gaming, and media processing. As a result, they deliver good performance across the workload range, but they fail to maximize either performance or efficiency on memory-intensive scale-out applications. Emerging server processors, on the other hand, employ simpler core microarchitectures that improve efficiency but fall short of maximizing performance. What the industry needs are server processors that jointly optimize for performance, energy, and TCO.
With this in mind, we developed a methodology for designing performance-densityoptimal server chips called Scale-Out Processors (SOPs). Our SOP methodology improves data-center efficiency through a many-core organization tuned to the demands of scale-out workloads.

\section{Today's server processors}

Multicore processors common today are well-suited for massively parallel scale-out workloads running in data centers. First, they improve throughput per chip over single-core designs. Second, they amortize on-chip and board-level resources among multiple hardware threads, thereby lowering both cost and power consumption per unit of work (that is, per thread).

Table 1 summarizes the principal characteristics of today's server processors. Existing data centers are built with server-class designs from Intel and AMD. A representative processor is Intel's Xeon $5670,{ }^{4}$ a mid-range design that integrates six powerful dualthreaded cores and a spacious 12-Mbyte last-level cache (LLC). The Xeon 5670 consumes $95 \mathrm{~W}$ at a maximum frequency of $3 \mathrm{GHz}$. The combination of powerful cores and relatively large chip size leads us to classify conventional server processors as big-core, big-chip designs.

Recently, several companies have introduced processors featuring simpler core microarchitectures that specifically target 


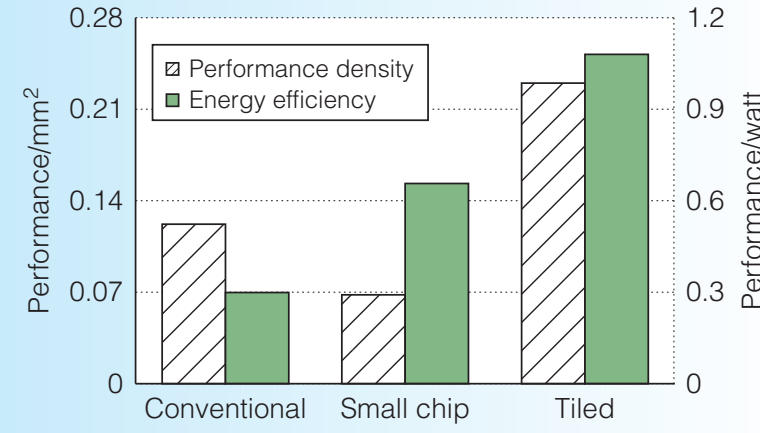

(a)

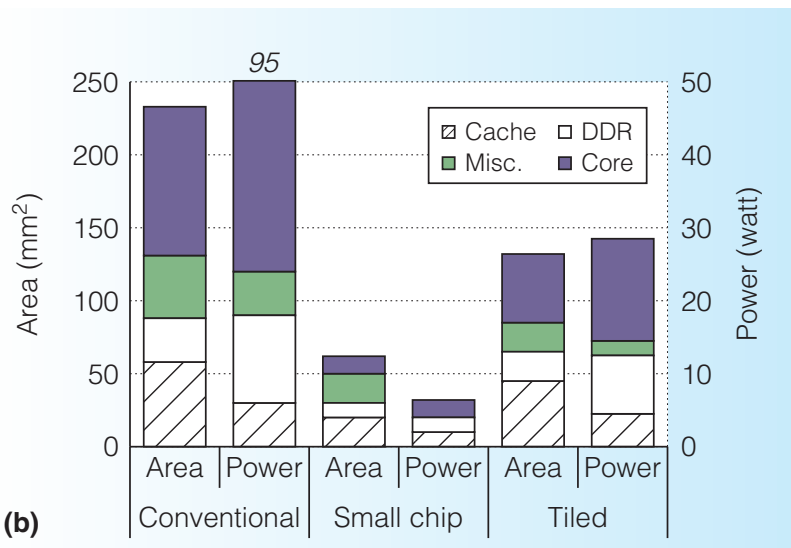

Figure 1. Efficiency, area, and power of today's server processors: performance density and energy efficiency (a); processor area and power breakdown (b). We use a combination of analytic models and simulation-based studies to estimate the performance, area, and power characteristics.

scale-out data centers. Research has shown simple-core designs to be well-matched to the demands of many scale-out workloads, which spend a high fraction of their time accessing memory and have moderate computational intensity. ${ }^{5}$ Two design paradigms have emerged in this space: one type features a few small cores on a small chip (small core, small chip); the other integrates many small cores on a bigger chip (small core, big chip).

Companies including Calxeda, Marvell, and SeaMicro market small-core, small-chip processors targeted at data centers. Despite the differences in the core organization and even the instruction set architecture (ISA)_Calxeda's and Marvell's designs are powered by ARM, whereas SeaMicro uses an x86-based Atom processor-the chips are surprisingly similar in their feature set: all have four hardware contexts, dual-issue cores, a clock speed in the range of 1.1 to 1.6 $\mathrm{GHz}$, and power consumption of 5 to $10 \mathrm{~W}$. We use the Calxeda design as a representative configuration, featuring four Cortex-A9 cores, a 4-Mbyte LLC, and an on-die memory controller. ${ }^{6}$ At $1.5 \mathrm{GHz}$, our model estimates a peak power consumption of $6 \mathrm{~W}$.

A processor representative of the smallcore, big-chip design philosophy is Tilera's Tile-Gx3036. This server-class processor features 36 simple cores and a 9-Mbyte LLC in a tiled organization. ${ }^{7}$ Each tile integrates a core, a slice of the shared LLC, and a router. Accesses to the distributed LLC's remote banks require a traversal of the on-chip interconnect, implemented as a $2 \mathrm{D}$ mesh network with a single-cycle per-hop delay. Operating at $1.5 \mathrm{GHz}$, the Tilera-like tiled design draws approximately $28 \mathrm{~W}$ of power at peak load.

To understand the efficiency implications of these diverse processor architectures, we use a combination of analytic models and simulation-based studies, employing a fullsystem server simulation infrastructure, to estimate their performance, area, and power characteristics. Our workloads are taken from CloudSuite (http://parsa.epfl.ch/cloudsuite), a collection of representative scale-out applications that includes web search, data serving, and MapReduce.

Figure 1a compares the designs along two dimensions: performance density and energy efficiency. Performance density, expressed as performance per $\mathrm{mm}^{2}$, measures the processor's ability to effectively utilize the chip real estate. Energy efficiency, in units of performance per watt, indicates the processor's ability to convert energy into useful work.

The small-core, small-chip processor offers a $2.2 \times$ improvement in energy efficiency over a conventional big-core design, thanks to the former's simpler core microarchitecture. However, the small-chip design has 45 percent lower performance density than the conventional one. To better 
understand the trends, Figure $1 \mathrm{~b}$ shows a breakdown of the respective processors' area and power budgets. The data reveals that while the cores in the conventional server processor take up 44 percent of the chip area, the small-chip design commits just 20 percent of the chip to compute, with the remainder of the area going to the LLC, $\mathrm{I} / \mathrm{O}$, and auxiliary circuitry. In terms of power, the six conventional cores consume $71 \mathrm{~W}$ of the $95-\mathrm{W}$ power budget $(75$ percent), whereas the four simpler cores in the small-chip organization dissipate just $2.4 \mathrm{~W}$ (38 percent of total chip power) under full load. As with the area, the relative energy cost of the cache and peripheral circuitry in the small-chip design is greater than in the conventional design (62 percent and 25 percent of the respective chips' power budgets).

The most-efficient design point is the small-core, big-chip tiled processor, which surpasses both conventional and small-chip alternatives by more than 88 percent in performance density, and 65 percent in energy efficiency. The cores in the tiled processor take up 36 percent of the chip real estate, nearly doubling the fraction of the area dedicated to execution resources as compared to the small-chip design. The fraction of the power devoted to execution resources increases to 48 percent compared to 38 percent in the small-chip design.

Our results corroborate earlier studies that identify efficiency benefits stemming from the use of lower-complexity cores as compared to those used in conventional server processors. ${ }^{8,9}$ However, our findings also identify an important, yet unsurprising, trend: the use of simpler cores by themselves is insufficient for maximizing processor efficiency, and the chip-level organization must be considered. More specifically, a larger chip that integrates many cores is necessary to amortize the area and power expense of uncore resources, such as cache and off-chip interfaces, by multiplexing them among the cores.

\section{Scale-Out Processors}

To maximize silicon efficiency on scaleout workloads, we examined the characteristics of a suite of representative scale-out applications and the demands they place on processor resources. Our findings, consistent with prior work, ${ }^{10,11}$ indicate that

- large LLCs are not beneficial for capturing data-center applications' enormous data footprint;

- the active instruction footprint greatly exceeds the Level-1 (L1) caches' capacity, but can be accommodated with a 2- to 4-Mbyte secondary cache; and

- scale-out workloads have virtually no thread-to-thread communication, requiring minimal on-chip coherence and communication infrastructure.

Driven by these observations, we developed the SOP design methodology that extends the small-core, big-chip design space by optimizing the on-chip cache capacity, core count, interconnect delay, and number of interfaces to the off-chip memory in a way that maximizes computing density and throughput. ${ }^{12}$

At the heart of an SOP is a coarse-grained building block called a pod-a stand-alone multicore server. Each pod features a modestly sized 2- to 4-Mbyte LLC for capturing the active instruction footprint and commonly accessed data structures. The small LLC size reduces the cache access time and leaves more chip area for the cores. To further reduce the latency of performancecritical LLC accesses, SOPs use a highbandwidth crossbar interconnect instead of a multihop point-to-point network. The number of cores in a pod is empirically chosen in a way that maximizes cache utilization without causing thrashing or penalizing interconnect area and delay.

The SOP architecture achieves scalability through tiling at the pod granularity up to the available area, power, or memory bandwidth limit. The multiple pods share the off-chip interfaces to reduce cost and maximize bandwidth utilization. The pod-based tiling strategy reduces chip-level complexity and provides a technology-scalable architecture that preserves each pod's optimality across technology generations. Figure $2 \mathrm{com}$ pares the SOP chip architecture to conventional, small-chip, and tiled designs.

Compared to a tiled design, an SOP increases the number of cores integrated on 


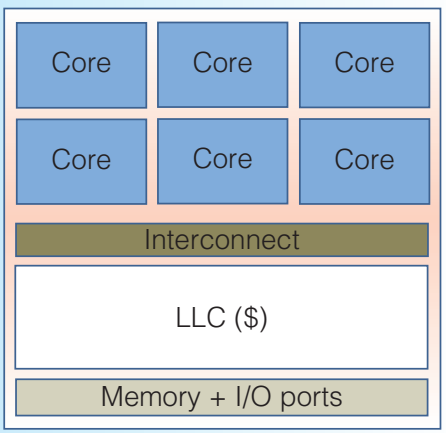

(a)

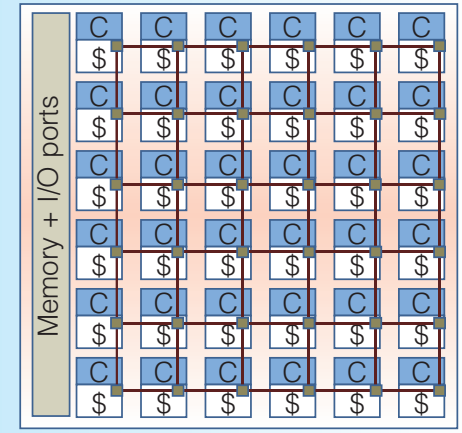

(c)

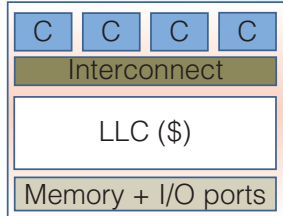

(b)

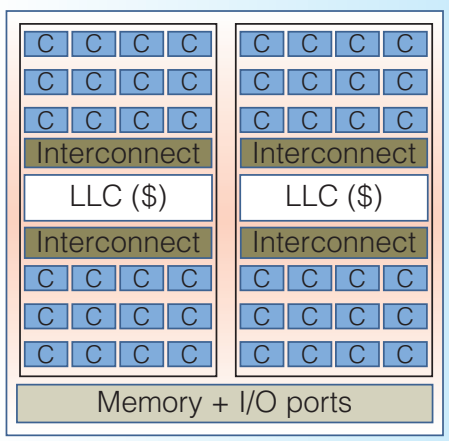

(d)

Figure 2. Comparison of server processor organizations: conventional (a), small chip (b), tiled (c), and Scale Out (d). The Scale-Out design achieves the highest performance density through modestly sized caches and many cores in a multi-pod organization.

chip by 33 percent, from 36 to 48, within the same area budget. SOPs achieve this computing-density improvement by reducing the LLC capacity from 9 to 4 Mbytes, freeing up chip area for the cores. The resulting SOP devotes 47 percent of the chip area to the cores, up from 36 percent in the tiled processor. Our evaluation shows that the percore performance in the SOP design is comparable to that in a tiled one; although the SOP's smaller LLC capacity has a dampening effect on single-threaded performance, the lower access delay in the crossbar-based SOP compensates for the higher miss ratio by accelerating fetches of performancecritical instructions. The bottom line is that the SOP design improves chip-level performance (that is, throughput) by 33 percent over the tiled processor. Finally, the SOP design's peak power consumption is higher than that of the tiled processor owing to

the former's greater on-chip computing capacity. However, as our results demonstrate, the SOP's greater chip-level processing capability is beneficial from a TCO perspective despite the increased power draw at the chip level.

\section{Methodology}

We now describe the cost models, server hardware features, workloads, and simulation infrastructure used in evaluating the various chip organizations at the data-center level.

\section{TCO model}

Large-scale data centers employ highdensity server racks to reduce the space footprint and improve cost efficiency. A standard rack can accommodate up to $421 \mathrm{U}$ (one-rack-unit) servers, with each server integrating one or more processors, multiple DRAM DIMMs, disk- or flash-based storage nodes, and a network interface. Servers in a rack share the power distribution infrastructure and network interfaces with the rest of the data center. The number of racks in a large-scale data center is commonly constrained by the available power budget.

Our TCO analysis, derived using EETCO, ${ }^{13}$ considers four major expense categories. Table 2 further details the key parameters.

Data-center infrastructure. This includes the land, building, and power provisioning and cooling equipment with a 15 -year depreciation schedule. The data-center area is primarily determined by the IT (rack) area, with cooling and power provisioning equipment factored in. We estimate the cost of this equipment per watt of critical power.

Server and networking hardware. Server hardware includes processors, memory, disks, and motherboards. We also account for the networking gear at the data center's edge, aggregation, and core layers and assume that the cost scales with the number of racks. The amortization schedule is three years for server hardware, and four years for networking equipment.

Power. This is predominantly determined by the servers, including fans and power 
Table 2. Total cost of ownership (TCO) parameters.

\begin{tabular}{ll} 
Parameter & Value \\
\hline Rack dimensions (42U): width $\times$ depth $\times$ inter-rack & $0.6 \mathrm{~m} \times 1.2 \mathrm{~m} \times 1.2 \mathrm{~m}$ \\
$\quad$ space & \\
Infrastructure cost & $\$ 3,000 / \mathrm{m}^{2}$ \\
Cooling and power provisioning equipment cost & $\$ 12.5 / \mathrm{W}$ \\
Cooling and power provisioning equipment space & $20 \%$ \\
$\quad$ & \\
$\quad$ overhead & 1.3 \\
SPUE (server power usage effectiveness) factor & 1.3 \\
PUE (data-center power usage effectiveness) factor & $\$ 200$ per rack/month \\
Personnel cost & $360 \mathrm{~W}, \$ 10,000$ per rack \\
Networking gear & $25 \mathrm{~W}, \$ 330$ per rack unit \\
Motherboard & $10 \mathrm{~W}, \$ 180,100$-year mean time to \\
Disk & failure (MTTF) \\
& $1 \mathrm{~W}, \$ 25,800$-year MTTF per Gbyte \\
DRAM & 30 -year MTTF \\
Processor &
\end{tabular}

supplies, networking gear, and cooling equipment. The electricity cost is $\$ 0.07 / \mathrm{KWh}$.

Maintenance. This includes costs for repairing faulty equipment, determined by its mean time to failure (MTTF), and the salaries of the personnel.

\section{Server processors}

We evaluate a number of data-center server processor designs, as summarized in Table 1 . We use publicly available data from the open web and Microprocessor Report (http://www.mpronline.com/index.php) to estimate core and chip area, power, and cost. We supplement this information with Cacti 6 for cache area and power profiles, and we use measurements of commercial server processors' die micrographs to estimate the area of on-chip memory and I/O interfaces. We detail many aspects of the methodology in our International Symposium on Computer Architecture (ISCA) 2012 paper. $^{12}$

Baseline parameters. To make the analysis tractable and reduce variability due to differences in the ISA, we model ARM-based cores for all but the conventional processor designs. We based both the tiled and SOP in-order configurations on the Cortex-A8, a dual-issue in-order core clocked at $1.5 \mathrm{GHz}$. The small-chip design uses a more aggressive dual-issue out-of-order core, similar to an ARM Cortex-A9, which is representative of existing products in the smallchip processor design space. For the conventional design, we model a custom four-wide large-window core running at $3 \mathrm{GHz}$.

At the chip level, we model the target processors' associated cache configurations, interconnect topologies, and memory interfaces. Our simulations reflect important runtime artifacts of these structures, such as interconnect delays, bank contention, and off-die bandwidth limitations.

Effect of higher-complexity cores. Although the low-complexity out-of-order and inorder cores are attractive from an area- and energy-efficiency perspective, their lower performance could be unacceptable for applications that demand fast response times and have nontrivial computational components $\left(\right.$ for example, web search ${ }^{8}$ ). To study the effect of higher-performance cores on datacenter efficiency, we also evaluate an SOP organization based on an ARM CortexA15, a triple-issue core clocked at $2 \mathrm{GHz}$. Compared to the baseline dual-issue inorder core, a three-way out-of-order core delivers 81 percent higher single-threaded performance, on average, across our workload suite, while requiring $3.5 \times$ the area and more than $3 \times$ the power per core. 
Effect of chip size. The results in Figure 1 suggest that small-core designs on a larger chip improve performance density and energy efficiency as compared to small-chip organizations. To better understand the effect of chip size on data-center efficiency, we extend the evaluation space of tiled and SOP processors with additional designs featuring twice the core, cache, and memory interfaces. These " $2 \times$ " designs approximately match the area of the Xeon-based conventional processor considered in this study.

Processor price estimation. We estimated the price for the conventional processor by picking the lowest price $(\$ 800)$ among online vendors for the target Xeon 5670 processor. Prices for tiled (\$300) and small-chip (\$95) designs are sourced from the November 2011 Microprocessor Report and correspond to Tilera Tile-Gx3036 and Calxeda ECX1000 , respectively.

To compute the cost for the various SOP and $2 \times$ tiled designs, we used the Cadence InCyte Chip Estimation tool. We estimated the production volume of the Tilera Gx3036 processor to be 200,000 units, given a selling price of $\$ 300$ and a 50 -percent margin. We used this production volume to estimate each processor type's selling price, considering nonrecurring engineering (NRE) costs, mask and production costs, yield, other expense categories, and a 50percent profit margin. Although we used these estimates for the majority of the studies, we also considered the sensitivity of different designs to processor price.

\section{Workloads and simulation infrastructure}

We took our workloads, which included Data Serving, MapReduce, SAT Solver, Web FrontEnd, and Web Search, from CloudSuite. For the Web FrontEnd workload, we used the e-banking option from SPECweb2009 in place of its open-source counterpart from CloudSuite, because SPECweb2009 exhibits better performance scalability at high core counts. Functionally, all these applications have similar characteristicsnamely, they operate on huge data sets that are split across a large number of nodes into memory-resident shards, the nodes service a large number of completely independent requests that don't share state, and the internode connectivity is used only for high-level task management and coordination. ${ }^{11}$ Two of the workloads-SAT Solver and MapReduce - are batch, whereas the rest are latency sensitive and are tuned to meet the response-time objectives.

We estimated the various processor designs' performance using the Flexus fullsystem simulation. ${ }^{14}$ Our performance metric is the product of UIPC (a ratio of committed user instructions over the sum of both user and system cycles) and processor frequency. UIPC is a more accurate performance metric in full-system evaluation than total IPC due to the contribution of I/O and spin locks in the operating system to the execution time. ${ }^{14}$

Because of space constraints, we only present aggregate results across all workloads. We averaged performance using a harmonic mean.

\section{Experimental setup}

For all experiments, we assume a fixed data-center power budget of $20 \mathrm{MW}$ and a power limit of $17 \mathrm{~kW}$ per rack. We evaluated lower-density racks rated at $6.6 \mathrm{~kW}$, but we found the trends to be similar across the two rack configurations. Therefore, for space considerations, we present only one set of results.

To compare the different server architectures' performance and TCO, we start with a rack power budget and subtract all power costs at both the rack and board level, excluding the processors. The per-rack costs include network gear, cooling (fans), and power conversion. At the $1 \mathrm{U}$ server level, we account for the motherboard, two disks, and memory (model parameter) power. The remaining power budget is divided by each evaluated processor chip's peak power to determine the number of processors per server. We then estimate data-center performance as the product of per-processor performance (using the data collected in simulation) and the number of processors in each $1 \mathrm{U}$ server, the number of servers in a rack, and the number of racks in the data center.

Finally, we make no assumptions about what the optimal amount of memory per 
server is, which in practice varies for different workloads. Instead, we model three server configurations-with 32, 64, and 128 Gbytes of memory per 1U. One simplifying assumption we make is that the amount of memory per $1 \mathrm{U}$ is independent of the chip design. Underlying this assumption are the observations that the data is predominantly read-only, and is partitioned for high parallelism, allowing performance to scale with more cores and sockets until bottlenecked by the bandwidth of the memory interfaces. Our studies account for bandwidth limitations.

\section{Evaluation}

We examined the various processor designs' performance and TCO, relative efficiency, and sensitivity to processor price.

\section{Performance and TCO}

We first compare data-center performance and TCO for various processor designs, assuming 64 Gbytes of memory per $1 \mathrm{U}$ server. Figure 3 presents the results.

In general, we observe significant disparity in data-center performance across the processor range, stemming from the different capabilities and energy profiles of the various processor architectures. Highly integrated processors based on small cores, namely tiled and SOP, deliver the highest performance at the data-center level. The tiled small-core, big-chip architecture improves aggregate performance by a factor of 3.6 over conventional and 1.6 over small-chip designs. The tiled design is superior, thanks to a combination of efficient core microarchitectures and high chip-level integrationattributes that help amortize the power of both chip- and node-level resources among many cores, affording more power for execution resources.

The highest performance is delivered by the SOP with in-order cores, a small-core, big-chip design with the highest performance density, which improves data-center performance by an additional 10 percent over the tiled processor. The SOP design effectively translates its performance-density advantage into a performance advantage by better amortizing fixed-power overheads among its many cores, ultimately affording more
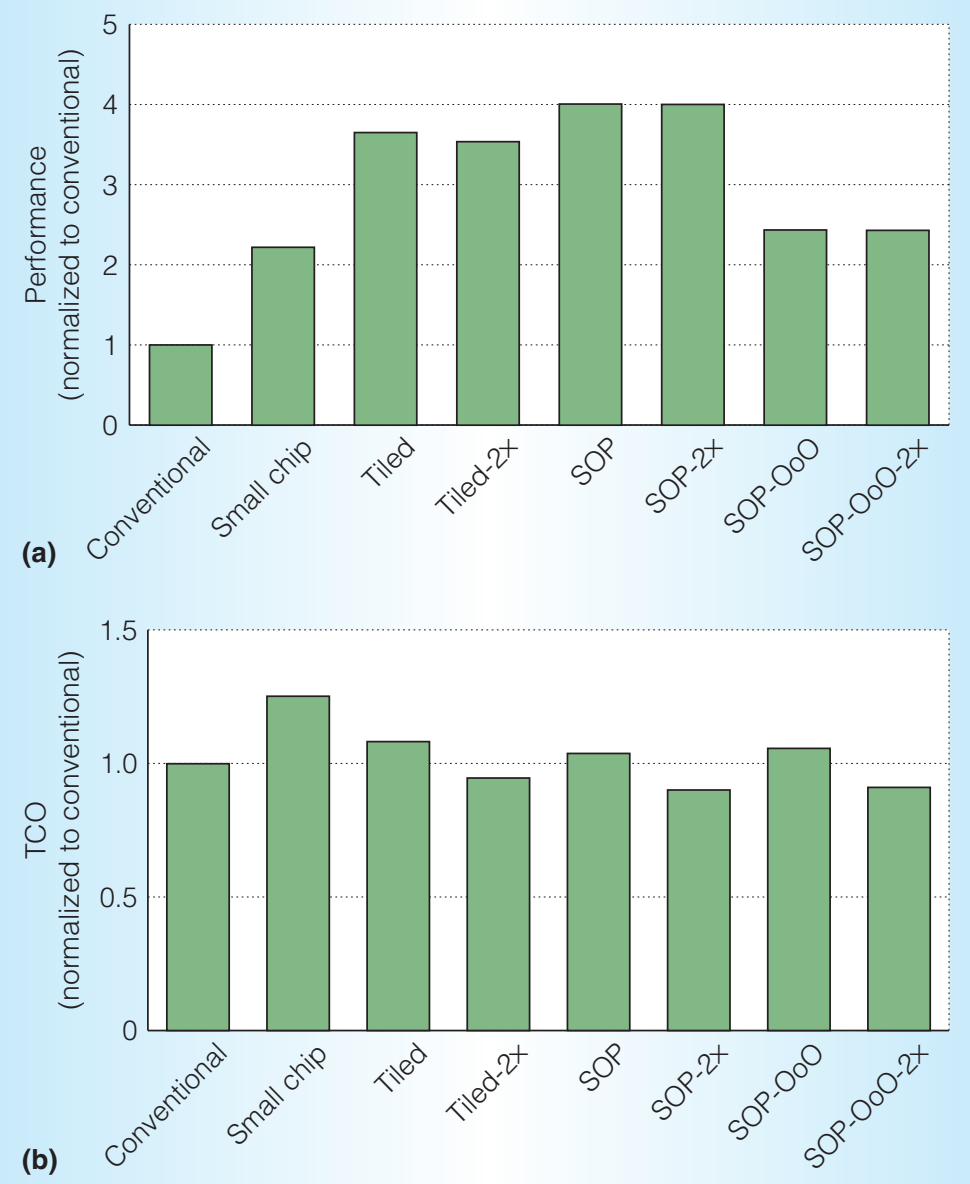

Figure 3. Data-center performance and TCO for various server processors normalized to a design based on the conventional processor: data-center performance (higher is better) (a); data-center TCO (lower is better) (b). Differences in processors' organizations result in large performance differences at the data-center level. Meanwhile, processor choice has a modest effect on data-center TCO as other costs dominate.

power for the execution resources at the rack level.

The SOP design based on out-of-order cores sacrifices 39 percent of the throughput at the data-center level compared to the inorder design. However, higher core complexity might be justified for workloads that demand tight latency guarantees and have a nontrivial computational component. Even with higher-complexity cores, the SOP architecture attains better data-center performance than either the conventional or small-chip alternatives.

The differences in TCO among the different designs aren't as pronounced as the 

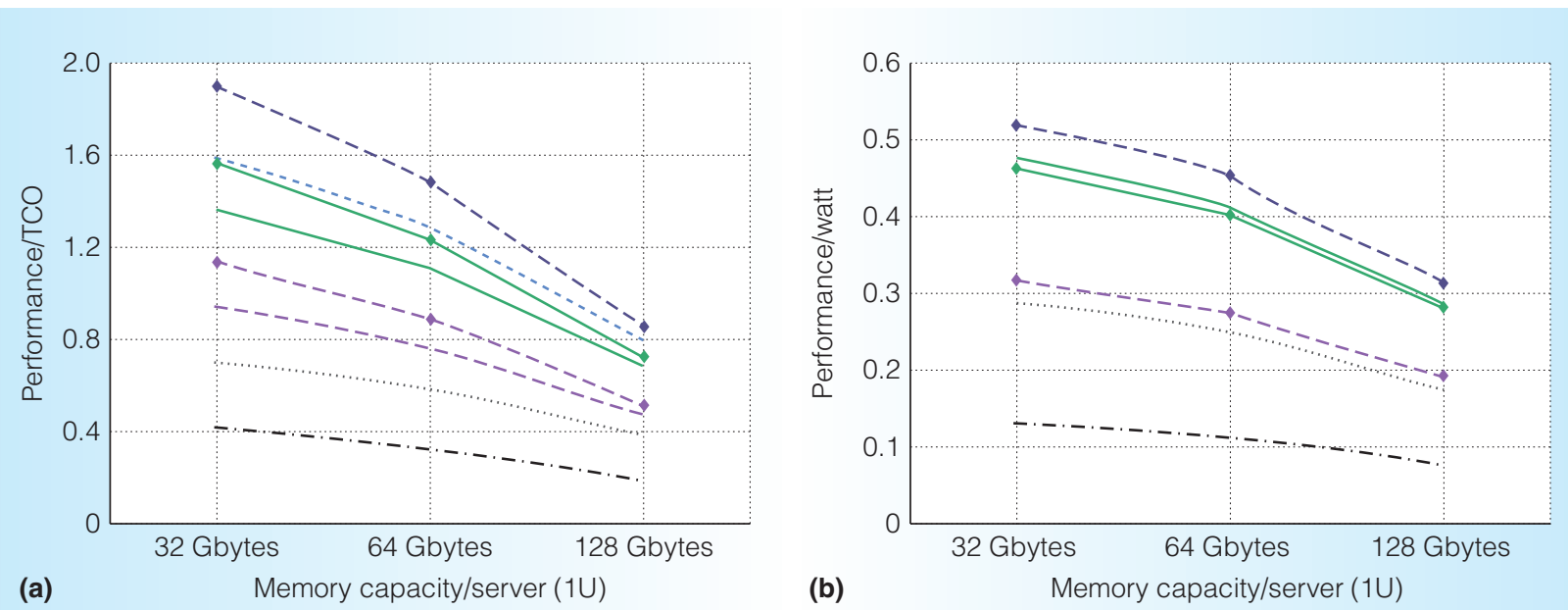

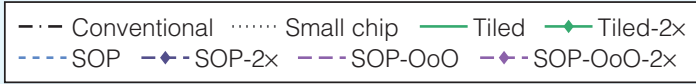

Figure 4. Data-center efficiency for different server processors: performance/TCO (a); performance/watt (b). Data is not normalized. Small-core, big-chip designs (that is, scale out and tiled) are the most efficient.

differences in performance, because processors contribute only a fraction (19 to 39 percent) to the overall data-center acquisition and power budget. Nevertheless, one important trend worth highlighting is that although small-chip designs are significantly less expensive and more energy efficient (by a factor of 8 and 2.2, respectively) than conventional processors on a per-unit basis, a small-chip design has a 25 percent higher TCO at the data-center level. The reason for this apparent paradox is that the small-chip design's limited computing capabilities necessitate as many as 32 sockets per $1 \mathrm{U}$ server (versus 2 for conventional) to saturate the available power budget. The acquisition costs of such a large number of chips negate the differences in unit price and energy efficiency, emphasizing the need to consider TCO in assessing datacenter efficiency.

\section{Relative efficiency}

We next examine the combined effects of performance, energy efficiency, and TCO by assessing the various designs on data-center performance/TCO and performance/watt. Figure 4 presents the results as memory capacity varies from 32 to 128 Gbytes per $1 \mathrm{U}$ server.
With 64 Gbytes of memory per $1 \mathrm{U}$ server, we observe the following trends:

- The small-chip design improves performance/watt by $2.2 \times$ over the conventional processor, but its performance/ TCO advantage is just $1.8 \times$ due to the high processor acquisition costs.

- A data center based on the tiled design improves performance per TCO by a factor of 3.4 over conventional and 1.9 over small-chip designs. Energy efficiency is improved by $3.6 \times$ and $1.6 \times$, respectively, underscoring the combined benefits of aggressive integration and an efficient core microarchitecture.

- SOP designs with an in-order core further improve performance/TCO by 14 percent and performance/watt by 10 percent over tiled processors through a more efficient use of chip real estate.

- Tiled and SOP designs with twice the resources (Tiled- $2 \times$ and SOP- $2 \times$ ) improve TCO by 11 to 15 percent over their baseline counterparts by reducing the number of processor chips, thereby lowering acquisition costs.

- The SOP design with out-of-order cores achieves 40 percent lower performance/TCO than the design based 
on in-order cores. The more aggressive out-of-order microarchitecture is responsible for each core's lower energy and area efficiency, resulting in lower throughput at the chip level. When the TCO premium is justified, which might be the case for profit-generating latency-sensitive applications (such as web search), the out-of-order SOP design offers a $2.3 \times$ performance $/ \mathrm{TCO}$ advantage $(2.4 \times$ in performance/watt) over the conventional server processor.

Although the earlier discussion focuses on servers with 64 Gbytes of memory, the trends are similar with other memory configurations. In general, servers with more memory lower the performance-to-TCO ratio, as memory adds to the server cost while diminishing the processor power budget. The opposite is true for servers with less memory, in which the choice of processor has a greater effect on both cost and performance.

The key result of our study-that highly integrated server processors are beneficial from a TCO perspective-is philosophically similar to the observations made by Karidis et al., who noted that high-capacity servers are effective in lowering the cost of computation. $^{15}$

\section{Sensitivity to processor price}

Figure 5 shows the effect of varying the processor price on the relative efficiency (performance/TCO) of the different designs, assuming 64 Gbytes of memory per $1 \mathrm{U}$ server. For each processor type, except conventional, we assume an application-specific integrated circuit (ASIC) design in 40-nm technology, and we compute the price as a function of market size, ranging from $40 \mathrm{~K}$ to $2 \mathrm{M}$ units. For the conventional design, we linearly sweep the price from $\$ 800$ (known market price) down to $\$ 200$, with the latter being the lowest considered price among area-equal Tiled- $2 \times$ and SOP- $2 \times$ designs.

In general, we observe that the price of larger chips has less impact on the datacenter TCO compared to that of smaller chips because it takes few large chips to populate a server due to power constraints. In contrast, the small-chip design is highly sensitive to unit price, owing to the sheer

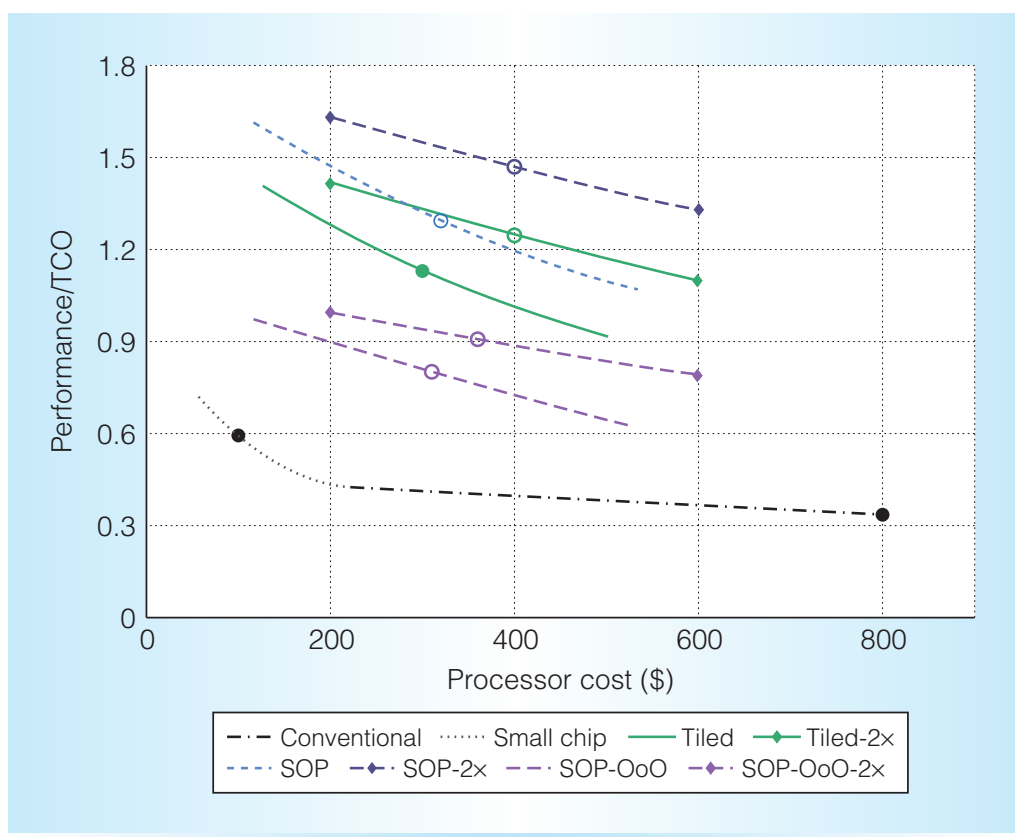

Figure 5. Relationship between the price per processor and the TCO. Solid circles indicate known prices; unfilled circles show estimated prices based on a production volume of $200 \mathrm{~K}$ units.

volume of chips required per $1 \mathrm{U}$ server. For instance, the respective numbers of chips per server in conventional versus small-chip designs differ by a factor of 16 .

A consistent trend in our study is that, from a TCO perspective, bigger chips are preferable to smaller ones, as seen in the curves for the various tiled and SOP designs. Although the larger chip area of the " $2 \times$ " designs adds expense, the price difference is modest (around 16 percent or $\$ 50$ per chip), because NRE and design costs dominate production costs. Furthermore, the increased cost is offset by the reduction in the number of required chips.

S cale-Out Processors extend the advantages of emerging small-core, big-chip architectures, providing good energy efficiency via simple core microarchitectures, and maximizing the TCO investment by fully utilizing server hardware via abundant execution resources. In the near term, SOPs will be able to deliver performance and TCO gains in a nondisruptive manner by fully leveraging existing software stacks. Further out, demands for greater performance and energy efficiency could necessitate 
even higher degrees of specialization, requiring a disruptive change to the programming model. Our ongoing research effectively targets both near- and long-term data-center efficiency challenges through integration, specialization, and approximation-the new "ISA" of the 21 st century.

\section{Acknowledgments}

We thank Sachin Idgunji and Emre Ozer, both affiliated with ARM during the work described here, for their contributions to the article. We thank the EuroCloud project partners for inspiring the Scale-Out Processors. This work was partially supported by EuroCloud, project 247779 of the European Commission 7th RTD Framework ProgrammeInformation and Communication Technologies: Computing Systems.

\section{References}

1. U. Hölzle and L.A. Barroso, The Datacenter as a Computer: An Introduction to the Design of Warehouse-Scale Machines, Morgan and Claypool Publishers, 2009.

2. SAVVIS, "SAVVIS Sells Assets Related to Two Data Centers for \$200 Million," June 2007.

3. J. Hamilton, "'Overall Data Center Costs," blog, 18 Sept. 2010; http://perspectives.mvdirona. com/2010/09/18/OverallDataCenterCosts. aspx.

4. T.P. Morgan, "Facebook's Open Hardware: Does It Compute?" The Register, 8 Apr. 2011; http://www.theregister.co.uk/2011/ 04/08/open_compute_server_comment, 2011.

5. M. Berezecki et al., "Many-Core Key-Value Store," Proc. Int'l Green Computing Conf. and Workshops, IEEE CS, 2011, doi:10.1109/ IGCC.2011.6008565.

6. Calxeda, "Calxeda EnergyCore ECX-1000 Series," May 2012; http://www.calxeda.com/ wp-content/uploads/2012/06/ECX1000Product-Brief-612.pdf.

7. Tilera, "TILE-Gx 3036 Specifications," 2011, http://www.tilera.com/sites/default/files/ productbriefs/Tile-Gx\%203036\%20SB01201.pdf.

8. V.J. Reddi et al., "Web Search Using Mobile Cores: Quantifying and Mitigating the Price of Efficiency," Proc. 37th Ann. Int'l Symp.
Computer Architecture (ISCA 10), ACM, 2010, pp. 314-325.

9. K. Lim et al., "'Understanding and Designing New Server Architectures for Emerging Warehouse-Computing Environments," Proc. 35th Ann. Int'l Symp. Computer Architecture (ISCA 08), IEEE CS, 2008, pp. 315-326.

10. N. Hardavellas et al., "Toward Dark Silicon in Servers," IEEE Micro, July/Aug. 2011, pp. 6-15.

11. M. Ferdman et al., "Clearing the Clouds: A Study of Emerging Scale-Out Workloads on Modern Hardware," Proc. 17th Int'l Conf. Architectural Support for Programming Languages and Operating Systems (ASPLOS 12), ACM, 2012, pp. 37-48.

12. P. Lotfi-Kamran et al., "Scale-Out Processors," Proc. 39th Ann. Int'l Symp. Computer Architecture (ISCA 12), IEEE CS, 2012, pp. 500-511.

13. D. Hardy et al., "EETCO: A Tool to Estimate and Explore the Implications of Datacenter Design Choices on the TCO and the Environmental Impact," Proc. 1st Workshop Energy-Efficient Computing for a Sustainable World, 2011; http://eurocloudserver. com/wp-content/uploads/EESC2011.pdf.

14. T. Wenisch et al., "SimFlex: Statistical Sampling of Computer System Simulation," IEEE Micro, July/Aug. 2006, pp. 18-31.

15. J. Karidis, J.E. Moreira, and J. Moreno, "True Value: Assessing and Optimizing the Cost of Computing at the Data Center Level," Proc. 6th ACM Conf. Computing Frontiers, ACM, 2009, pp. 185-192.

Boris Grot is a postdoctoral researcher at EPFL (École Polytechnique Fédérale de Lausanne). His research interests include processor architectures, memory systems, and interconnection networks for highthroughput, energy-aware computing. Grot has a $\mathrm{PhD}$ in computer science from the University of Texas at Austin. $\mathrm{He}$ is a member of IEEE and the ACM.

Damien Hardy is a postdoctoral researcher at the University of Cyprus. His research interests include computer architecture, reliability, embedded and real-time systems, and data center modeling. Hardy has a PhD in computer science from the University of Rennes, France. 
Pejman Lotfi-Kamran is a fifth-year $\mathrm{PhD}$ candidate at EPFL. His research interests include processor architecture and interconnection networks for high-throughput and energy-efficient data centers. Lotfi-Kamran has an MSc in computer architecture from the University of Tehran. He is a member of IEEE and the ACM.

Babak Falsafi is a professor of computer and communication sciences at EPFL, and the founding director of EcoCloud, an interdisciplinary research center targeting robust, economic, and environmentally friendly cloud technologies. Falsafi has a $\mathrm{PhD}$ in computer science from the University of Wisconsin-Madison. $\mathrm{He}$ is a fellow of IEEE and a senior member of the ACM.

Chrysostomos Nicopoulos is a lecturer in the Department of Electrical and Computer Engineering at the University of Cyprus. His research interests include networks on chip
(NoCs), computer architecture, multi- and many-core microprocessor and system design, and architectural support for massively parallel computing. Nicopoulos has a $\mathrm{PhD}$ in electrical engineering from Pennsylvania State University. He is a member of IEEE and the ACM.

Yiannakis Sazeides is an associate professor in the Department of Computer Science at the University of Cyprus. His research focuses on computer architectures, particularly reliability, data-center modeling, memory hierarchy, temperature, and analysis of dynamic program behavior. Sazeides has a $\mathrm{PhD}$ in electrical engineering from the University of Wisconsin-Madison. He is a member of IEEE.

Direct questions and comments about this article to Boris Grot, EPFL IC ISIM PARSA, INJ 238 (Batiment INJ), Station 14, CH - 1015, Lausanne, Switzerland; boris.grot@epfl.ch.

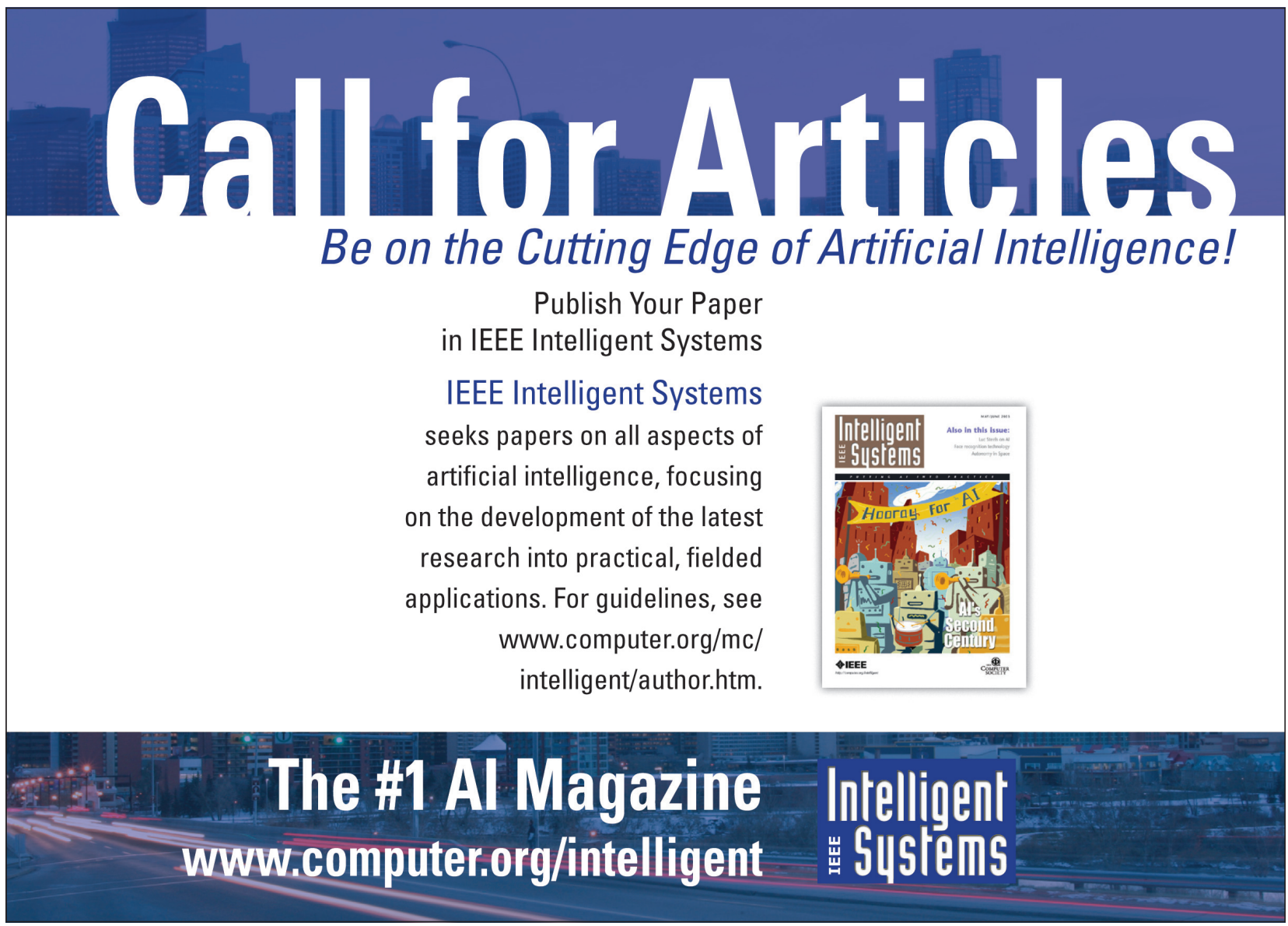

